\title{
ATRIBUTOS ANTECESSORES À SATISFAÇÃO E À CONFIANÇA NO SERVIÇO DE INTERNET BANKING
}

\author{
J. A. R. DE ARAÚJO ${ }^{1}$, T. H. DE S. ECHTERNACHT ${ }^{2}$, V. L. CRISÓSTOMO ${ }^{3}$, L. H. N. NOBRE ${ }^{4}$ \\ Universidade Federal Rural do Semi Árido ${ }^{1,4}$, Universidade Federal da Paraíba², Universidade Federal do Ceará ${ }^{3}$ \\ anizioaraujo@gmail.com ${ }^{1}$ \\ Submetido 14/08/2017 - Aceito 18/02/2020 \\ DOI: $10.15628 /$ holos. 2020.7116
}

\section{RESUMO}

Este artigo identifica os atributos que são antecedentes à geração de confiança e satisfação para os usuários dos serviços internet banking. Trata-se de uma pesquisa do tipo survey que contou com a participação de 449 respondentes brasileiros. Os dados foram tratados utilizando procedimento estatístico de sistematização dos dados, análise fatorial exploratória, validade de construto, construção de escalas, análise fatorial confirmatória e modelagem de equações estruturais. $O$ modelo teórico é formado por sete hipóteses sobre a relação entre confiança, satisfação, usabilidade, reputação e privacidade/segurança. No modelo proposto os construtos para confiança e satisfação aparecem como variáveis regressoras e apresentam um bom nível

de confiabilidade permitindo que seja observada a sua importância na prestação de serviços de internet banking. Os resultados sinalizam que níveis mais elevados de usabilidade, reputação e privacidade/segurança proporcionados pelos usuários deste serviço contribuem para melhores graus de confiança e de satisfação. A forma com que as pessoas veem o serviço de internet banking, ao comparar os produtos e serviços oferecidos por outros sites de bancos concorrentes, se relacionam positivamente com a confiança e satisfação depositada na prestação do serviço. Os resultados mostram também que a confiança dos usuários na utilização do serviço de internet banking não é um antecessor do grau de satisfação do mesmo.

Palavras-Chave: Satisfação; Confiança; Internet Banking

\section{ATTRIBUTES PREDECESSORS SATISFACTION AND CONFIDENCE IN THE INTERNET BANKING SERVICE}

\begin{abstract}
This article identifies the attributes that are antecedent to the generation of trust and satisfaction for users of internet banking services. This is a survey research that included the participation of 449 Brazilian respondents. The data were analyzed using statistical procedure of systematic data, exploratory factor analysis, construct validity, construction schedules, confirmatory factor analysis and structural equation modeling. The theoretical model consists of seven hypotheses about the relationship between trust, satisfaction, usability, reputation and privacy/security. In the model proposed constructs for confidence and satisfaction appear as
\end{abstract}

regression variables and have a good level of reliability allowing the observation of its importance in providing internet banking services. The results indicate that higher levels of usability, reputation and privacy/security provided by users of this service contribute to better levels of confidence and satisfaction. The way people see the internet banking service, to compare the products and services offered by other websites competing banks, is positively related to the trust and satisfaction deposited in providing the service. The results also show that the user confidence in the use of internet banking service is not a predecessor of the satisfaction of it.

Keywords: Satisfaction; Confidence; Internet Banking

\section{ATRIBUTOS SATISFACCIÓN PREDECESORES Y CONFIANZA EN EL SERVICIO DE BANCA POR INTERNET}

\section{RESUMEN}

Este artículo identifica los atributos que anteceden la generación de confianza y la satisfacción de los usuarios de los servicios de banca por Internet. El trabajo es una investigación tipo survey que contó con la participación de 449 encuestados brasileños. Los datos fueron analizados mediante el procedimiento estadístico de datos sistemáticos, análisis factorial exploratorio, la validez de constructo, los plazos de construcción, el análisis factorial confirmatorio y modelos de ecuaciones estructurales. El modelo teórico consta de siete hipótesis 
acerca de la relación entre la confianza, la satisfacción, la usabilidad, la reputación y la privacidad/seguridad. En el modelo propuesto los constructos para la confianza y satisfacción de aparecer como variables regresoras y tienen un buen nivel de fiabilidad que le permite observar su importancia en la prestación de los servicios de banca por Internet. Los resultados indican que los niveles más altos de la usabilidad, la reputación y la privacidad/seguridad proporcionados por los usuarios de este servicio contribuyen para mejorar los niveles de confianza y satisfacción. La forma en que la gente ve el servicio de banca por Internet, para comparar los productos y servicios ofrecidos por otros sitios web de los bancos de la competencia, se relaciona positivamente con la confianza y satisfacción depositado en la prestación del servicio. Los resultados también muestran que la confianza de los usuarios en el uso del servicio de banca por Internet no es un precursor de la satisfacción de la misma.

Palabras Clave: Satisfacción; la confianza; Banca por Internet

\section{INTRODUÇÃO}

A literatura dedicada ao marketing de relacionamento aponta o construto confiança como fator que favorece a redução da percepção de risco além de ser tradicionalmente considerado como elemento chave para a construção de relacionamentos de longo prazo, estáveis e rentáveis entre a empresa e seus clientes (Casaló, Carlos e Guinalíu 2011; Komiak e Benbasat, 2004; Ennew e Sekhon, 2007; Yousafzai, Foxall, e Pallister, 2010). Para Morgan e Hunt (1994), a confiança é apontada como fator central para as estratégias de marketing de relacionamento.

A confiança é resultado da interação de determinados atributos que a antecedem. Isto significa que a confiança não surge de forma espontânea, mas como resultado da interação de atributos que são percebidos pelo cliente como realidade. Gutman, Joia e Moreno Jr (2014) ressaltam que a confiança se refere à crença de que a promessa do outro será cumprida, mesmo em condições imprevistas. Assim, mesmo sendo relevante na intenção de adoção de um serviço de internet banking, ainda não se estabelece uma relação direta com a mesma. Na análise da intenção de uso de sistemas de home broker, a satisfação do usuário surge como como variável mediadora entre a confiança e a intenção de uso do sistema (Giovannini; Nunes; Ferreira, 2012; Gutman et al, 2014). Uma pesquisa de tecnologia bancária realizada pela Federação Brasileira de Bancos (FEBRABAN), revela que o consumidor tem mudado seus hábitos de utilização dos serviços bancários, fazendo com que os canais digitais (mobile banking e internet banking) apresente um crescimento da composição das transações bancárias de 47\% em 2014 para 60\% em 2018, quando comparados aos canais tradicionais e aos pontos de vendas no comércio (FEBRABAN, 2019).

Casaló et al. (2011) identificou os atributos reputação, percepção quanto a usabilidade, satisfação, privacidade e segurança, como relacionados à geração de confiança em ambientes virtuais. Estes atributos são coerentes com os principais fatores relacionados à adoção de tecnologias, oriundos de Modelo de Aceitação de Tecnologias (TAM) e destacados por Safeena, Kammani e Date (2014): facilidade de uso, privacidade e segurança, utilidade percebida, qualidade percebida, acessibilidade, confiança e satisfação. Assim, este estudo buscou investigar os fatores que contribuem para a geração de confiança e satisfação nos serviços de internet banking para o caso brasileiro. A questão proposta para esse trabalho pode ser assim descrita: Quais os atributos que favorecem a geração de confiança e satisfação nos usuários dos serviços de internet banking no Brasil? 
Hernandez e Mazzon (2008) realizaram um estudo relacional no Brasil sobre os determinantes para a adoção do internet banking, com usuários não brasileiros, onde além de variáveis ligadas ao indivíduo, utilizam variáveis relacionais como (vantagem relativa, visibilidade, demonstrabilidade de resultados, compatibilidade, complexidade, testagem, imagem). Outras pesquisas foram realizadas neste campo empírico no Brasil com outros objetivos, tais como delinear o perfil do usuário e a qualidade percebida do serviço (Santos, Conde, Santos e Antonialli, 2011); análise da relação entre a confiança, o valor percebido e a lealdade do usuário do internet banking (Brei e Rossi, 2005) e avaliação da qualidade de sites de internet banking sob a ótica dos seguintes fatores: facilidade de uso, a usabilidade e inovação, e a criatividade dos sites (Prado 2010). Assim, a relevância desse estudo repousa na premissa de que sendo a confiança e a satisfação fatores que surgem a partir de determinados atributos que os antecedem, então o conhecimento de tais atributos pode favorecer a adoção de estratégias que efetivamente permitam o estreitamento das relações entre a empresa e seus clientes. A confiança torna-se especialmente relevante em razão de os serviços de internet banking realizarem-se por meio de uma plataforma virtual, fato que eleva a percepção do cliente em relação ao risco, tornando a confiança fator indispensável para o sucesso deste tipo de canal de atendimento.

O artigo está estruturado da forma que se segue. Além desta introdução a seção 2 apresenta a revisão de literatura que fundamentam as hipóteses testadas no modelo. A seção 3 descreve os procedimentos metodológicos. A seção 4 com a análise de dados e discussão dos resultados e a seção 5 conclui.

\section{REVISÃO DE LITERATURA}

\subsection{INTERNET BANKING COMO FERRAMENTA PARA O MARKETING DE RELACIONAMENTO}

O marketing de relacionamento pode ser compreendido como o esforço integrado da empresa para identificar, construir, manter e aprimorar relacionamentos individuais com clientes, em um processo de troca de benefícios voltado para o longo prazo (Berry, 2002). Tal esforço integra, portanto, um conjunto de ações voltadas à construção de vínculos emocionais com os clientes, à tentativa de conhecimento mais aprofundado dos consumidores e ao desenvolvimento de confiança e comprometimento entre as partes (Berry, 2002; O'Malley e Tynan, 2000). Nesse esforço, a ferramenta internet banking pode contribuir para o estreitamento das relações entre os clientes e o banco.

Devido a sua criticidade em termos de segurança observa-se que a plataforma internet banking somente será efetiva à medida em que for capaz de satisfazer e conquistar a confiança de seu usuário (Twum e Ahenkora, 2012). Morgan e Hunt (1994), na tentativa de buscar uma definição a respeito do que seria central para o conceito de marketing de relacionamento, concluíram que deve ser algo que distinga trocas relacionais produtivas e efetivas das improdutivas e não efetivas. Nesse sentido, ainda que muitos outros fatores possam contribuir para o sucesso ou fracasso de um relacionamento específico, Morgan e Hunt (1994) defendem que a confiança e o comprometimento são conceitos centrais e essenciais para o sucesso de um marketing de relacionamento.

Moutinho e Smith (2000) relatam evidência na literatura sobre serviços financeiros que confirmam a hipótese de que acesso e conveniência são dois critérios dominantes para escolha da 
instituição financeira e subsequente satisfação. Nesse contexto, os serviços de internet banking proporcionam tanto acesso facilitado quanto conveniência, sendo fator que pode efetivamente contribuir como ferramenta para o marketing da empresa junto a seus clientes. Sikdar, Kumar e Makkad (2015) corroboram com esta ideia e reforçam que as plataformas online redefiniram a interface do relacionamento dos clientes com as entidades bancárias. Estas relações, depois de fortalecidas, geram benefícios mais difíceis de serem replicados e proporcionam elementos para um relacionamento de sucesso de longo prazo (Morgan-Thomas e Veloutsou, 2013).

2.2 Atributos necessários à geração de confiança e satisfação nos serviços de internet banking

A literatura apresenta diferentes definições para o conceito do construto confiança. Para Rousseau, Sitkin, Burt e Camerer (1998, p.395) a "confiança é um estado psicológico que compreende a intenção de aceitar uma vulnerabilidade baseada em expectativas positivas das intenções ou comportamentos de outro". Grönroos (2000, p.37) define a confiança como "a expectativa de uma parte de que a outra parte se comportará de uma maneira previsível em uma dada situação". Outra definição usualmente citada é a "tendência a acreditar num parceiro de negócios no qual se tem crédito" (Moorman, Deshpandé e Zaltman, 1993, p. 82).

A confiança é um dos atributos apontados como chave para o sucesso das atividades que ocorrem em meio virtual, tais como os serviços de internet banking (Yu, Balaji e Khong, 2015; Kesharwani e Bisht, 2012; Zhu e Chen, 2012; Zhao, Lewis, Hanmer-Lloyd e Ward, 2010; Jarvenpaa, Tractinsky e Vitale, 2000). Jarvenpaa et al (2000) identificou relações causais bastante expressivas entre a confiança e as transações em meio digital, tendo em vista que a confiança é fator que reduz o risco percebido. A construção da confiança nas transações online, segundo Casaló et al. (2011) e Bart, Shankar, Sultan e Urban (2005) é análoga à que se desenvolve por uma loja física, e como resultado, o consumidor desenvolve a percepção de confiança com base em suas interações com a loja física ou com o website. No entanto, em meio virtual, a falta da presença física e o anonimato inerentes ao meio fazem com que as percepções dos clientes sobre as empresas sejam ainda mais importantes (Loebbecke \& Hornig, 2001). Hanson (2000) identificou que a falta de confiança tem sido apontada como um dos fatores que desmotivam as transações em meio virtual.

A satisfação pode ser definida como o resultado da avaliação global de todos os aspectos que envolvem a relação entre a empresa e seu consumidor (Casaló et al., 2011; Viacava et al., 2015). O uso da satisfação do usuário como fator determinante para a adoção de sua tecnologia foi iniciado por Bailey e Pearson (1983), e disseminado como pode ser visto pela ampla literatura direcionada ao tema: Ives, Olson e Baroudi (1983), Melone (1990), Seddon (1997), Polatoglu e Ekin (2001), Wixom \& Todd, (2005); Gutman et al., (2014); Sikdar et al., (2015). No entanto, a inclusão da satisfação no Modelo de Aceitação de Tecnologia foi proposta por Wixom e Todd (2005). Considerando somente o setor financeiro, são recentes os estudos sobre a satisfação derivada de um serviço on-line como um elemento de comportamento de tomada de decisão do consumidor em ambientes virtuais (Sikdar et al., 2015). Algumas pesquisas estão voltadas a percepção dos clientes do internet banking quanto a sua satisfação na prestação do serviço (George, Kumar, 2015; Riquelme, Mekkaoui, Rios, 2009). Neste estudo, consonante com o trabalho de Gutman et al., (2014), optou-se por adotar a satisfação do usuário com o sistema de internet banking, como 
uma variável proxy da atitude do cliente em relação ao uso do sistema. Susanto, Chang e Há, (2016), encontraram evidências de que a satisfação do usuário em serviços de internet banking é impactada significativamente pela confiança e pela utilidade dos usuários.

Nessa perspectiva, torna-se relevante identificar os fatores associados à construção da confiança e satisfação no ambiente virtual, mais especificamente em relação aos serviços de internet banking. Tomando como base o trabalho de Casaló et al. (2011), o presente estudo buscou testar os atributos apontados como geradores de confiança para os usuários de serviços de internet banking que atuam no Brasil. Os atributos testados foram: Confiança, Satisfação, Usabilidade; Reputação, Privacidade e Segurança. Por fim, testou-se a relação entre as variáveis do estudo: confiança e satisfação.

\subsubsection{Percepção quanto à Usabilidade}

Por usabilidade compreende-se a facilidade de navegação no website. Para Casaló et al. (2011) a usabilidade é fator que influencia a confiança em razão de alguns fatores como: i) a maior usabilidade reduz a probabilidade de erros por parte do usuário, o que tende a elevar sua confiança (Muir \& Moray, 1996); ii) a maior usabilidade percebida torna o usuário mais confiante em si mesmo e em usar o website (Kantowitz, Hankowski e Kantowitz, 1997); iii) maior usabilidade percebida proporciona uma atmosfera de maior conforto, o que favorece o uso do website e as transações online (Corritore, Kracher e Wiedenbeck, 2003). Liébana-Cabanillas, Muñoz-Leiva, Rejón-Guardia (2013) confirmaram as hipóteses de que a facilidade do uso do serviço bancário eletrônico de uma instituição financeira, determinam positivamente a satisfação e a confiança no serviço prestado. Desse modo, é plausível sugerir as seguintes hipóteses:

Hipótese 1: A usabilidade percebida no internet banking tem efeito positivo sobre a satisfação de seu usuário.

Hipótese 2: A usabilidade percebida no internet banking tem efeito positivo sobre a confiança do cliente no fornecedor do serviço.

\subsubsection{Percepção quanto à Reputação}

A reputação é um conceito diretamente associado à credibilidade percebida pelos consumidores. Casaló et al. (2011) propõe que a reputação pode ser o resultado entre o que a empresa promete e aquilo que ela efetivamente entrega, ou seja, a reputação é construída à medida em que a empresa cumpre os compromissos firmados com seus clientes e em como ela lida com eles. Assim, no contexto das transações virtuais, a reputação pode ser uma medida coletiva de confiança, que tem por base as avaliações dos seus consumidores (Josang, Ismail \& Boyd, 2007). Tendo em vista que as transações em meio virtual se caracterizam por dispensar o contato físico, a reputação da instituição prestadora do serviço pode ser um importante fator que contribui para elevar a confiança do usuário dos serviços de internet banking (Casaló et al., 2011). Estes fatores motivam a proposição da seguinte hipótese:

Hipótese 3: Um mais elevado nível de reputação percebida tem efeito positivo sobre a confiança no fornecedor do serviço.

Também é oportuno a sugestão da hipótese de que a reputação adquirida pela empresa proprietária do website tenha implicação direta no grau de percepção da satisfação por parte de seus clientes como sugerido na hipótese 4 uma vez que a reputação do prestador de serviço pode 
ser capaz de deixar o cliente mais satisfeito ao sentir-se provido por uma empresa que goza de bom histórico no mercado.

Hipótese 4: Um mais elevado nível de reputação percebida tem efeito positivo sobre a satisfação do usuário do internet banking.

\subsubsection{Percepção quanto à Segurança e Privacidade}

Um dos principais obstáculos para o desenvolvimento das transações virtuais são os problemas relacionados à possível falta de privacidade e segurança em relação às informações enviadas por meio da Internet. Kolsaker e Payne (2002) sugerem que a percepção dos consumidores acerca da segurança nos meios de pagamento e nos mecanismos de transmissão dos dados é necessária para o desenvolvimento da confiança no ambiente virtual. Gerrard, Cunningham e Devlin (2006) identificaram fatores que explicam a distância que certos clientes mantêm do internet banking. Os mais críticos são o risco percebido e a não necessidade, ou seja, se há algum risco de perda de privacidade e há um método tradicional seguro o cliente preferirá o canal mais seguro. A preocupação dos usuários da internet com aspectos relacionados a sua privacidade vem sendo analisada intensamente como a percepção global de risco em ambientes virtuais (Liao et al 2011; Li, 2014). Privacidade e segurança têm sido compreendidos como dois conceitos que têm uma relação muito próxima. Para Flavián e Guinalíu (2006), privacidade e segurança são dimensões de um único constructo, que pode ser compreendido como a segurança percebida no tratamento de dados privados. Ao sentir segurança o cliente tem mais confiança na privacidade de suas transações e torna-se mais satisfeito como proposto nas seguintes hipóteses.

Hipótese 5: Um maior nível de percepção na privacidade e segurança no que diz respeito ao tratamento dos dados pessoais dos consumidores tem influência positiva sobre a satisfação percebida pelo usuário do internet banking.

Hipótese 6: Um maior nível de percepção na privacidade e segurança no que diz respeito ao tratamento dos dados pessoais dos consumidores tem efeito positivo sobre a confiança no fornecedor do serviço.

Por se tratar de uma construção complexa e multifacetada a confiança não tem um consenso para uma definição (Ibrahim; Noor; Mehad, 2009). Enquanto construto é utilizada em inúmeras pesquisas relacionando-se com outras variáveis. Alguns estudos como Butt, Aftab (2013), Casaló et al. (2011), Bauer, Grether, Leach (2002) e Selnes (1998), apontam a satisfação como atributo antecessor à confiança, outros estudos como Liébana-Cabanillas et al (2013), Ma (2012), Gupta \& Bansal (2012), Roostika (2011) e Liao \& Cheung (2008) consideram a confiança como um antecedente a satisfação na utilização do serviço de internet banking, valorizando desse modo uma preocupação mundial que está voltada para superar as vulnerabilidades existentes na utilização deste serviço online. Para Khan \& Rahman (2017) a satisfação do consumidor é uma síntese avaliativa de sua experiência de consumo, que se fundamenta na diferença entre a expectativa anterior e o desempenho real percebido após consumo. Ofori et al. (2017) também testa a hipótese de que a confiança se relaciona positivamente com a satisfação para clientes do internet banking, eles afirmam que é mais provável que um consumidor que confie em uma marca esteja satisfeito e disposto a se comprometer com essa marca. 
Em relação aos construtos confiança e satisfação, espera-se que haja uma relação positiva entre o nível de confiança percebido e a satisfação por parte do usuário como proposto na hipótese 7.

\section{Hipótese 7: A confiança no fornecedor do serviço tem efeito positivo sobre a satisfação do usuário do internet banking.}

A Figura 1 resume o modelo estrutural que irá testar as hipóteses para as variáveis latentes utilizadas.

Figura 1 - Modelo Estrutural das Variáveis Latentes proposto na pesquisa

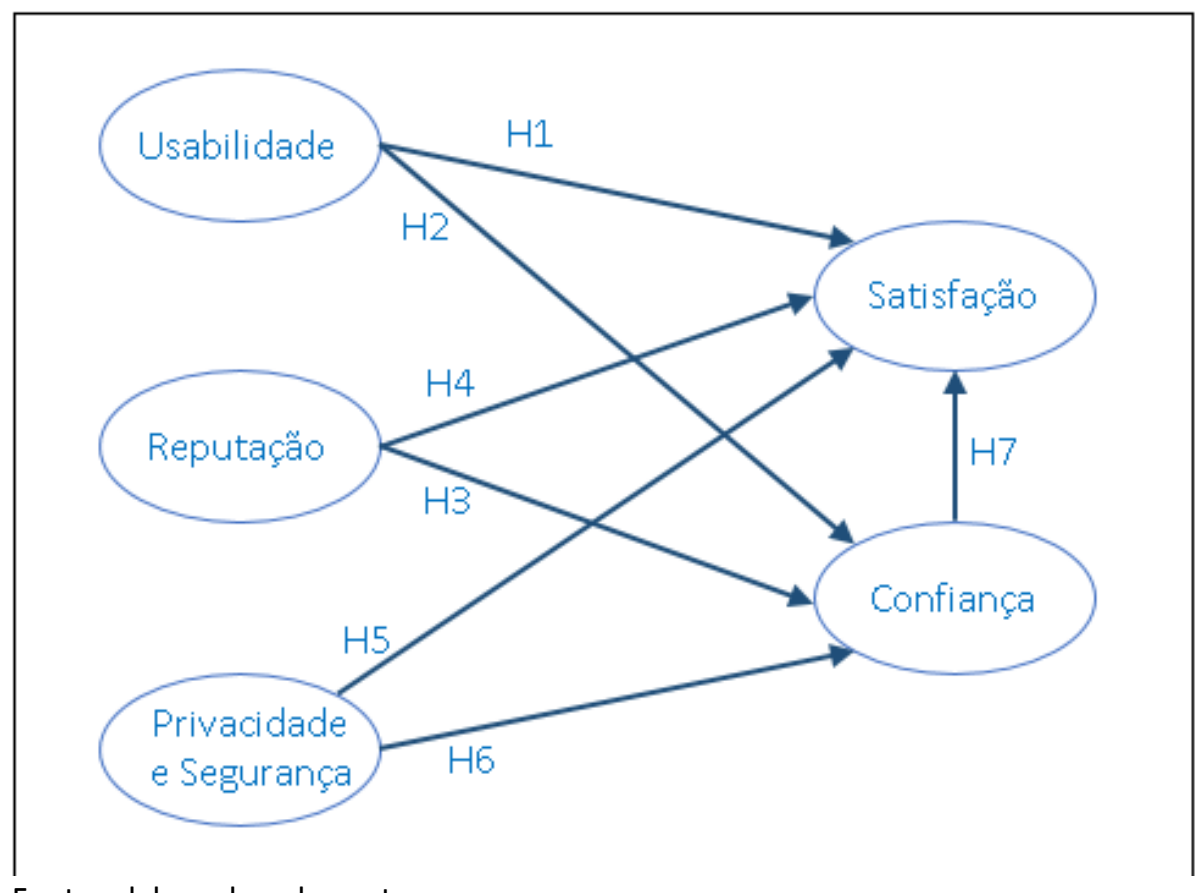

Fonte: elaborado pelos autores

\section{METODOLOGIA}

Este estudo é de natureza aplicada, com objetivo exploratório e descritivo, utiliza abordagem survey, sem controle de variáveis, com abrangência intencional, análise quantitativa e temporalidade cross section. A amostra foi por conveniência e todos os respondentes declararam ser usuários dos serviços de internet banking. A coleta dos dados ocorreu por meio de questionários padronizados aplicados a membros da comunidade universitária, em três campi de duas universidades. O questionário utilizado na pesquisa utilizou escala de Likert de 7 pontos e foi auto respondido pelos participantes. O instrumento de pesquisa foi adaptado de Casaló et al. (2011), que examina os antecedentes da confiança do consumidor nos serviços e na distribuição de produtos online, contendo alguns ajustes para identificar os atributos que são antecedentes à geração de confiança e satisfação para os usuários dos serviços de internet banking. A análise estatística foi realizada com o uso dos softwares Statistical Package for Social Science (SPSS), versão 21.0 (SPSS Inc, Chicago, IL, EUA) e AMOS (v.21, SPSS Inc, Chicago, IL, EUA). 


\section{RESULTADOS EMPÍRICOS}

\subsection{Caracterização da Amostra}

A pesquisa contou com a participação de 449 respondentes brasileiros que após rigoroso processo de exclusão de missing values e outliers multivariados, resultou em uma amostra com 407 questionários considerados válidos. Inicialmente foi realizada a sistematização dos dados, a estatística descritiva, assimetria, curtose e normalidade. Em seguida a amostra foi dividida de modo estocástico em duas partes (partição $A, \operatorname{com} n=204$ e partição $B, \operatorname{com} n=203$ ). $A$ partição $B$ foi utilizada para trabalhar a análise fatorial exploratória e verificação do modelo de equações estruturais. A outra parte da amostra (partição A) foi utilizada para estabelecer a avaliação da qualidade do ajustamento do modelo teórico à estrutura correlacional observada e à validade convergente de cada construto, por meio da análise fatorial confirmatória, análise de correlações, variância média extraída e da confiabilidade composta.

Na caracterização da amostra, observou-se a presença de indivíduos de diversas classes sociais, econômicas e culturais. Destaque-se que $48,6 \%$ dos participantes utilizam o serviço de internet banking há mais de dois anos e apenas $27,3 \%$ é usuário deste serviço há menos de um ano. Os respondentes foram em sua maioria do sexo masculino com $62,4 \%$, sendo apenas $37,6 \%$ do sexo feminino. Os respondentes apresentam um perfil jovem, sendo 48,7\% com idade até 24 anos e $35,1 \%$ com idade entre 25 e 34 anos. Destaque-se o grau de escolaridade, sendo $55,8 \%$ dos participantes alunos de graduação, $15,7 \%$ com nível superior completo e $27,3 \%$ detentores de título de pós-graduação. A Tabela 1 apresenta a amostra seccionada por banco em que os respondentes operam utilizando o serviço do internet banking, estratificando os indivíduos por gênero e a renda familiar.

Tabela 1 - Distribuição percentual cruzada dos Bancos em relação ao gênero e a renda familiar

\begin{tabular}{ccccccccc}
\hline & \multicolumn{1}{c}{ Total } & \multicolumn{2}{c}{ Gênero } & \multicolumn{5}{c}{ Renda Familiar } \\
Banco & (\%) & Masc. & Fem. & $\begin{array}{c}\text { até 1 } \\
\text { SM }\end{array}$ & $\begin{array}{c}\text { de 1 a 3 } 3 \\
\text { SM }\end{array}$ & $\begin{array}{c}\text { de 3 a 6 } \\
\text { SM }\end{array}$ & $\begin{array}{c}\text { de 7 a 9 } \\
\text { SM }\end{array}$ & $\begin{array}{c}\text { acima de } \\
\text { 10 SM }\end{array}$ \\
\hline $\begin{array}{c}\text { Banco do } \\
\text { Brasil }\end{array}$ & $55,1 \%$ & $54,6 \%$ & $55,5 \%$ & $39,2 \%$ & $48,8 \%$ & $53,2 \%$ & $66,7 \%$ & $61,0 \%$ \\
Caixa & $27,0 \%$ & $28,3 \%$ & $24,8 \%$ & $30,4 \%$ & $32,8 \%$ & $22,6 \%$ & $21,8 \%$ & $29,2 \%$ \\
Bradesco & $8,4 \%$ & $7,5 \%$ & $9,8 \%$ & $26,1 \%$ & $6,7 \%$ & $13,9 \%$ & $1,3 \%$ & $4,2 \%$ \\
Itaú Unibanco & $3,9 \%$ & $2,8 \%$ & $5,9 \%$ & $4,3 \%$ & $4,2 \%$ & $4,3 \%$ & $5,1 \%$ & $1,4 \%$ \\
Banco Real & $0,7 \%$ & $0,8 \%$ & $0,7 \%$ & $0,0 \%$ & $0,8 \%$ & $1,7 \%$ & $0,0 \%$ & $0,0 \%$ \\
Santander & $4,4 \%$ & $5,5 \%$ & $2,6 \%$ & $0,0 \%$ & $5,0 \%$ & $4,3 \%$ & $5,1 \%$ & $4,2 \%$ \\
outro & $0,5 \%$ & $0,5 \%$ & $0,7 \%$ & $0,0 \%$ & $1,7 \%$ & $0,0 \%$ & $0,0 \%$ & $0,0 \%$ \\
\hline TOTAL & $100,0 \%$ & $100,0 \%$ & $100,0 \%$ & $100,0 \%$ & $100,0 \%$ & $100,0 \%$ & $100,0 \%$ & $100,0 \%$ \\
(contagem) & $(407)$ & $(254)$ & $(153)$ & $(23)$ & $(119)$ & $(115)$ & $(78)$ & $(72)$ \\
\hline
\end{tabular}

Nota: SM = R\$ 678,00; valor do salário mínimo em 2013; $(n=407)$

Fonte: Dados da pesquisa 2015

\subsection{Sistematização dos dados}

O instrumento de pesquisa está descrito na Tabela 2, o qual fez uso da escala de Likert de sete pontos, variando de 1 - discordo totalmente a 7 - concordo totalmente, sendo 4 o ponto de 
neutralidade (nem concordo, nem discordo). A Tabela 2 apresenta também a média, desvio padrão, assimetria (sk) e curtose (ku), calculadas para $n=407$. Os testes para normalidade de Kolmogorov-Smirnov e Shapiro-Wilk foram realizados na amostra apresentando resultado significativo para todas as variáveis (valor- $p<0,001)$. A ausência de normalidade multivariada verificada pelos testes é comum em amostras grandes que fazem uso da escala de Likert, por não ser uma escala intervalar. Segundo Marôco (2010), esses testes de ajustamento são extremamente sensíveis a pequenos desvios da normalidade, sendo elevada a probabilidade de ocorrência de erro do tipo I (concluir que a variável não se aproxima da distribuição normal, quando de fato sua distribuição é normal). Para Marôco (2010) valores de $|s k|<2$ e $|k u|<3$ não apresentam violação severa à distribuição normal, sendo, portanto, apropriados para a utilização do método Maximum Likelihood (ML). A Tabela 2 apresenta como valores absolutos máximos para assimetria (sk) de 1,11 e 1,94 para a curtose (ku), sendo adequado o uso do Maximum Likelihood na modelagem de equações estruturais.

Tabela 2 - Estatística Descritiva e Dimensões (DM)

\begin{tabular}{|c|c|c|c|c|c|c|}
\hline $\mathbf{Q}$ & Conteúdo & $\begin{array}{l}\text { Min- } \\
\text { Máx }\end{array}$ & $\begin{array}{l}\text { Média } \\
\text { (DP) }\end{array}$ & sk & ku & DM \\
\hline 1.1 & Neste site tudo é fácil de entender. & $2-7$ & $5,4(1,1)$ & $-1,01$ & 1,12 & \multirow{5}{*}{$\begin{array}{l}\text { ᄃ } \\
\frac{c}{0} \\
\frac{0}{0} \\
\overline{\bar{a}} \\
\frac{0}{0} \\
\frac{0}{0}\end{array}$} \\
\hline 1.2 & É fácil encontrar a informação que eu preciso neste site. & $2-7$ & $5,5(1,1)$ & $-0,94$ & 1,10 & \\
\hline 1.3 & A estrutura e o conteúdo deste site são fáceis de entender. & $2-7$ & $5,5(1,1)$ & $-0,82$ & 0,59 & \\
\hline 1.4 & É fácil navegar por esse site. & $2-7$ & $5,7(1,1)$ & $-1,08$ & 1,61 & \\
\hline 1.5 & $\begin{array}{l}\text { Quando estou navegando neste site, eu sinto que estou no controle } \\
\text { do que eu posso fazer. }\end{array}$ & $2-7$ & $5,4(1,2)$ & $-0,71$ & 0,36 & \\
\hline 2.1 & Vejo as pessoas falarem bem deste site. & $1-7$ & $4,8(1,3)$ & $-0,34$ & 0,28 & \multirow{4}{*}{ 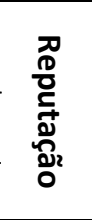 } \\
\hline 2.2 & $\begin{array}{l}\text { Comparado a outros sites de bancos concorrentes, este possui uma } \\
\text { boa reputação. }\end{array}$ & $1-7$ & $5,3(1,2)$ & $-0,29$ & $-0,54$ & \\
\hline 2.3 & Este site tem uma reputação de oferecer bons produtos e serviços. & $2-7$ & $5,3(1,0)$ & $-0,33$ & $-0,41$ & \\
\hline 2.4 & Este site é conhecido por ser justo na sua relação com seus usuários. & $1-7$ & $5,2(1,1)$ & $-0,32$ & 0,12 & \\
\hline 3.1 & Acho que tomei a decisão correta ao optar por este site. & $3-7$ & $6,0(0,9)$ & $-0,77$ & 0,14 & \multirow{4}{*}{ 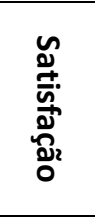 } \\
\hline 3.2 & A experiência que tenho tido com este site tem sido satisfatória. & $3-7$ & $6,1(0,9)$ & $-1,06$ & 1,46 & \\
\hline 3.3 & $\begin{array}{l}\text { Em termos gerais, estou satisfeito com a maneira que o site tem } \\
\text { realizado minhas operações bancárias. }\end{array}$ & $3-7$ & $6,0(1,0)$ & $-1,11$ & 1,23 & \\
\hline 3.4 & Em geral, estou satisfeito com o serviço que recebi do site. & $2-7$ & $5,9(0,9)$ & $-1,10$ & 1,94 & \\
\hline 4.1 & $\begin{array}{l}\text { Eu acho que as informações oferecidas neste site são sinceras e } \\
\text { honestas. }\end{array}$ & $2-7$ & $5,7(1,1)$ & $-0,86$ & 0,59 & \multirow{5}{*}{ 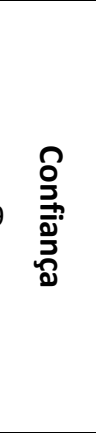 } \\
\hline 4.2 & $\begin{array}{l}\text { Este site é caracterizado pela franqueza e clareza dos serviços que ele } \\
\text { oferece para o consumidor. }\end{array}$ & $1-7$ & $5,3(1,2)$ & $-0,74$ & 0,49 & \\
\hline 4.3 & $\begin{array}{l}\text { Eu acho que este site se preocupa com os interesses presentes e } \\
\text { futuros de seus usuários. }\end{array}$ & $1-7$ & $5,1(1,1)$ & $-0,33$ & $-0,09$ & \\
\hline 4.4 & $\begin{array}{l}\text { Eu acho que este site possui as ferramentas necessárias para realizar } \\
\text { seu trabalho. }\end{array}$ & $2-7$ & $5,7(1,0)$ & $-0,80$ & 0,82 & \\
\hline 4.5 & $\begin{array}{l}\text { Eu acho que este site tem experiência suficiente na comercialização } \\
\text { dos produtos e serviços que ele oferece. }\end{array}$ & $2-7$ & $5,8(1,0)$ & $-0,57$ & 0,09 & \\
\hline 5.1 & $\begin{array}{l}\text { Acho que este site demonstra preocupação com a privacidade de seus } \\
\text { usuários. }\end{array}$ & $3-7$ & $6,1(1,0)$ & $-1,08$ & 0,62 & $\begin{array}{lll}0 & 0 \\
0 & 0 \\
0\end{array}$ \\
\hline \multicolumn{6}{|c|}{ HOLOS, Ano 36, v.1, e7116, 2020} & 9 \\
\hline
\end{tabular}


5.2 Eu me sinto seguro ao enviar informações pessoais para este site.

5.3 Acho que este site tem mecanismos que garantam a transmissão

5.3 segura de informações de seus usuários.

5.4 Ao enviar meus dados para este site, tenho certeza que não serão interceptados por terceiros não autorizados.

5.5 Acho que este site possui capacidade técnica suficiente para garantir 5.5 que os dados que envio não podem ser modificados por um terceiro.

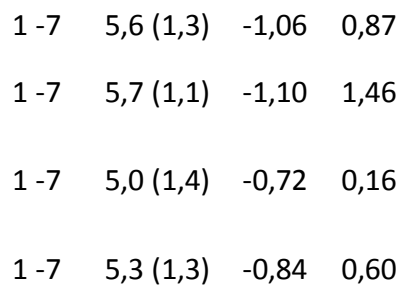

Fonte: Dados da pesquisa 2015, $(n=407)$

\subsection{Medidas de validação e resultados}

A Análise Fatorial Exploratória (AFE) foi utilizada para reduzir as variáveis diretamente observadas a um número de fatores adjacentes, descobrindo e analisando a estrutura de um conjunto de variáveis inter-relacionadas para se construir uma escala para fatores que, de alguma forma, controlam as variáveis originais. O método de extração utilizado para a obtenção dos fatores foi o da Análise dos Componentes Principais que considera a variância total dos dados, procurando uma combinação linear das variáveis observadas, de maneira a maximizar a variância total explicada (Fávero, Belfiore, Silva e Chan, 2009). O método de rotação utilizado foi o Varimax, que obtém uma estrutura fatorial na qual cada variável original esteja fortemente associada com os outros fatores (Marôco, 2011). A adequação da AFE foi testada por meio do teste KMO e o teste de esfericidade de Bartlett, com o propósito de saber se a matriz de correlação permite o uso da análise fatorial. A partição B da amostra, após a extração dos fatores, obteve valores do KMO de 0,659 a 0,823. Segundo Marôco (2011), valores de KMO entre 0,8 e 0,9 refletem uma boa recomendação quanto ao uso da análise fatorial. O teste de Bartlett mostrou-se significativo para todos os construtos indicando que a matriz de correlação apresenta correlações significantes entre pelo menos uma das variáveis (Hair, Tatham, Anderson e Black, 2009).

A Tabela 3 apresenta a síntese da AFE, com as cargas fatoriais, suas comunalidades, valor próprio (eigenvalue), variância explicada, KMO e Alfa de Cronbach para cada dimensão. As cargas fatoriais de cada dimensão são consideradas fortes variando de 0,604 a 0,879, bem como a comunalidade $\left(h^{2}\right)$ por apresentar valores acima de 0,5.

Tabela 3 - Síntese da Análise Fatorial Exploratória

\begin{tabular}{|c|c|c|c|c|c|c|c|}
\hline \multirow{2}{*}{ Dimensão } & \multirow{2}{*}{$\begin{array}{l}\text { Variável } \\
\text { Manifesta }\end{array}$} & \multicolumn{5}{|c|}{ Fatores } & \multirow{2}{*}{$h^{2}$} \\
\hline & & I & II & III & VI & v & \\
\hline \multirow{5}{*}{$\begin{array}{l}\text { Privacidade } \\
\text { e Segurança }\end{array}$} & q5_2 & $0,876 *$ & 0,048 & 0,148 & 0,156 & 0,082 & 0,822 \\
\hline & q5_4 & $0,860^{*}$ & 0,008 & 0,027 & $-0,020$ & 0,218 & 0,789 \\
\hline & q5_3 & $0,847^{*}$ & 0,057 & 0,155 & 0,182 & 0,003 & 0,778 \\
\hline & q5_5 & $0,846^{*}$ & 0,024 & 0,017 & $-0,018$ & 0,237 & 0,773 \\
\hline & q5_1 & $0,653^{*}$ & 0,116 & 0,249 & 0,171 & 0,028 & 0,531 \\
\hline \multirow{4}{*}{ Usabilidade } & q1_3 & 0,063 & $0,879 *$ & 0,106 & 0,147 & 0,098 & 0,820 \\
\hline & q1_2 & 0,008 & $0,802 *$ & 0,190 & 0,085 & 0,126 & 0,702 \\
\hline & q1_4 & 0,039 & $0,796 *$ & 0,098 & 0,221 & 0,098 & 0,704 \\
\hline & q1_1 & 0,102 & $0,783^{*}$ & 0,035 & 0,281 & 0,115 & 0,717 \\
\hline Reputação & q2_2 & 0,126 & 0,158 & $0,753^{*}$ & 0,101 & 0,060 & 0,621 \\
\hline
\end{tabular}




\begin{tabular}{|c|c|c|c|c|c|c|c|}
\hline & $q 2 \_1$ & 0,160 & 0,215 & 0,729* & 0,017 & 0,029 & 0,604 \\
\hline & $q 2 \_3$ & 0,120 & 0,064 & $0,720 *$ & 0,168 & 0,265 & 0,636 \\
\hline & $q 2 \_4$ & 0,073 & $-0,047$ & $0,633^{*}$ & 0,277 & 0,313 & 0,582 \\
\hline & q3_4 & 0,092 & 0,228 & 0,201 & $0,853^{*}$ & 0,170 & 0,856 \\
\hline Satisfação & q3_3 & 0,184 & 0,238 & 0,099 & $0,842^{*}$ & 0,120 & 0,823 \\
\hline & q3_2 & 0,115 & 0,283 & 0,185 & $0,770 *$ & 0,103 & 0,730 \\
\hline & q4_1 & 0,209 & 0,248 & $-0,011$ & 0,206 & $0,775^{*}$ & 0,748 \\
\hline Confiança & q4_2 & 0,192 & 0,180 & 0,347 & 0,038 & $0,717^{*}$ & 0,705 \\
\hline & q4_3 & 0,113 & 0,058 & 0,339 & 0,150 & $0,604^{*}$ & 0,519 \\
\hline $\begin{array}{l}\text { Valor } \\
\text { (Eigenvalue) }\end{array}$ & Próprio & 3,556 & 2,925 & 2,349 & 2,438 & 1,908 & \\
\hline Variância Exp & & $71,12 \%$ & $73,12 \%$ & $58,72 \%$ & $81,25 \%$ & $63,59 \%$ & \\
\hline KMO & & 0,814 & 0,823 & 0,742 & 0,724 & 0,659 & \\
\hline Alfa de Cronk & & 0,896 & 0,877 & 0,757 & 0,884 & 0,713 & \\
\hline
\end{tabular}

Fonte: Dados da pesquisa 2015, utilizando a partição $B$ da amostra $(n=203)$.

*Cargas fatoriais significativas. $h^{2}$ - Comunalidade;

Método de extração: ACP; Método de Rotação: Varimax com normalização de Kaiser;

Segundo Hair et al. (2009, p.587): "a análise fatorial confirmatória (AFC) é uma maneira de testar o quão bem as variáveis medidas representam os construtos", permitindo verificar a estrutura latente do modelo, buscando indicadores de qualidade de ajuste. Os construtos obtidos por meio da AFE foram validados por meio do método Maximum Likelihood (ML) na AFC com o uso da partição $A$ da amostra. O método ML é a função de ajuste mais amplamente utilizada em equações estruturais, sendo assintoticamente não viciados, consistentes e normalmente distribuídos (Marôco, 2010; Hair et al., 2009). A avaliação da validade dos construtos utilizados no modelo estrutural é apresentada na Tabela 4 pelo Alfa de Cronbach, Variância Média Extraída e a Confiabilidade Composta. O Alfa de Cronbach é o coeficiente mais comum para a análise da confiabilidade dos dados e tem como idéia principal a de que os indicadores individuais devem medir os mesmos construtos a serem inter correlacionados (Lee e Hooley, 2005; Kline, 2000). A Variância Média Extraída (VME) indica se o percentual de variação explicada dos indicadores é grande o suficiente para garantir a qualidade de mensuração. A Confiabilidade Composta (CC) difere do Alfa de Cronbach por não fazer suposição de que os itens são oriundos do mesmo domínio em que as correlações médias entre os indicadores são uma constante. Para Hair et al. (2009) o limite inferior geralmente aceito para o Alfa de Cronbach para a Confiabilidade Composta (CC) é 0,7 e valores acima de 0,5 para a Variância Média Extraída (VME) indicam uma convergência adequada.

Tabela 4 - Avaliação da validade global das medidas dos construtos

\begin{tabular}{cccc}
\hline Construtos & $\begin{array}{c}\text { Alfa de } \\
\text { Cronbach }\end{array}$ & $\begin{array}{c}\text { Variância Média } \\
\text { Extraída }\end{array}$ & $\begin{array}{c}\text { Confiabilidade } \\
\text { Composta }\end{array}$ \\
\hline Privacidade e Segurança & 0,890 & 0,678 & 0,892 \\
Usabilidade & 0,879 & 0,697 & 0,881 \\
Reputação & 0,779 & 0,569 & 0,785
\end{tabular}




$\begin{array}{llll}\text { Satisfação } & 0,876 & 0,717 & 0,861 \\ \text { Confiança } & 0,704 & 0,563 & 0,724\end{array}$

Fonte: Dados da pesquisa 2015, utilizando a partição $A$ da amostra $(n=204)$.

A Tabela 5 apresenta a correlação entre os construtos. Pode-se verificar a capacidade de discriminação entre as dimensões dos componentes do e-service. As correlações devem apresentar valores inferiores a 0,95, para assegurar a não sobreposição de conceitos e medidas entre os construtos da análise, sendo classificadas como moderadas ao apresentar valores entre 0,25 e 0,50; e forte com valores entre 0,50 e 0,75 (Hair et al. 2009; Marôco 2011). O construto confiança está fortemente correlacionado com Privacidade/Segurança, Reputação e Satisfação. A variável latente Usabilidade apresenta uma correlação moderada em relação aos construtos Privacidade/Segurança, Reputação e Confiança.

Tabela 5 - Correlação entre os construtos

\begin{tabular}{cccccc}
\hline & $\begin{array}{c}\text { Privacidade e } \\
\text { Segurança }\end{array}$ & Usabilidade & Reputação & Satisfação Confiança \\
\hline Privacidade e Segurança & 1 & & & & \\
Usabilidade & 0,286 & 1 & & & \\
Reputação & 0,542 & 0,364 & 1 & & \\
Satisfação & 0,520 & 0,608 & 0,453 & 1 & 1 \\
Confiança & 0,608 & 0,435 & 0,778 & 0,664 & 1 \\
\hline
\end{tabular}

Nota: as correlações são significativas com valor-p<0,001

Fonte: Dados da pesquisa 2015, utilizando a partição $A$ da amostra $(n=204)$.

A Tabela 6 apresenta as medidas de ajuste geral utilizadas como parâmetros de avaliação do modelo AFC.

Tabela 6 - Medidas de ajuste geral do modelo AFC

\begin{tabular}{|c|c|c|}
\hline Medida & Valores de referência* & $\begin{array}{c}\text { Valor } \\
\text { calculado** }\end{array}$ \\
\hline$x^{2}-$ Chi-squared & & 238,538 \\
\hline $\begin{array}{c}x^{2} / g l-\text { Chi-squared por graus de } \\
\text { liberdade }\end{array}$ & $\begin{array}{c}>5 \text { - ajustamento mau } \\
\text { ]2, 5] - ajustamento sofrível } \\
\text { ]1, 2] - ajustamento bom } \\
\sim 1 \text { - ajustamento muito bom }\end{array}$ & 1,716 \\
\hline $\begin{array}{c}\text { SRMR - Standardized Root Mean Square } \\
\text { Residual (I. C. 95\%) }\end{array}$ & $\begin{array}{c}>0,10-\text { ajustamento pobre } \\
\text { ]0,08; 0,10] - ajustamento medíocre } \\
\text { ]0,05; 0,08] - ajustamento aceitável } \\
\leq 0,05 \text { - bom ajustamento }\end{array}$ & 0,056 \\
\hline GFI - Goodness of Fit Index & $<0,8-$ ajustamento mau & 0,898 \\
\hline NFI - Normed Fit Index & {$[0,8 ; 0,9[-$ ajustamento sofrível } & 0,898 \\
\hline CFI - Comparative Fit Index & {$[0,9 ; 0,95[-$ ajustamento bom } & 0,954 \\
\hline TLI - Tucker-Lewis Index & $\geq 0,95$ - ajustamento muito bom & 0,943 \\
\hline AGFI - Adjusted Goodness of Fit Index & $=1-$ ajuste perfeito & 0,860 \\
\hline $\begin{array}{l}\text { RMSEA - Root Mean Square Error of } \\
\text { Approximation (I. C. 95\%) }\end{array}$ & $\begin{array}{l}>0,10-\text { ajustamento inaceitável } \\
\text { ]0,05;0,10] - ajustamento bom } \\
\leq 0,05-\text { ajustamento muito bom }\end{array}$ & 0,059 \\
\hline PGFI - Parsimony Goodness of Fit Index & $<0.6$ - ajustamento mau & 0,657 \\
\hline
\end{tabular}




$\begin{array}{ccc}\text { PCFI - Parsimony Comparative Fit Index } & \begin{array}{c}{[0,6 ; 0,8[- \text { ajustamento bom }} \\ \geq 0,8-\text { ajustamento muito bom }\end{array} & 0,775\end{array}$

Fonte: *Marôco (2010), Hair et al. (2009), Kline (2005), ${ }^{* *}$ Dados da pesquisa 2015, com resultados significativos a 0,001 .

$\mathrm{O}$ ajustamento do modelo foi realizado por meio dos índices comparativos RMSEA, CFI, GFI e PCFI. Os erros (e1-e2, e8-e11 e e11-e12) tiveram suas cargas balanceadas dentro do mesmo construto, utilizando os valores mais elevados dos índices de modificação das covariâncias. 0 número de variáveis manifestas utilizadas no modelo $(m=19)$, associado ao tamanho da partição A da amostra $(n=204)$ e considerando a estatística Chi-squared $=238,538$ (valor- $p<0,001), g \mid=$ 139; $\mathrm{CFI}=0,954 ;$ SRMR =0,056 e RMSEA = 0,059, indicam, uma boa qualidade de ajuste (Hair et al. 2009).

4.4 O modelo proposto e verificação das hipóteses

O Modelo de Equações Estruturais é utilizado para estabelecer relações entre as variáveis do estudo e permite testar as hipóteses apresentadas na Figura 1 . O ajustamento do modelo utilizou os índices CFI e GFI (valores superiores a 0,9) e PCFI e PGFI (valores superiores a 0,6) (Marôco, 2010). A Figura 3 apresenta o output do modelo de equações estruturais com as estimativas padronizadas.

Figura 3 - Modelo Estrutural (valores padronizados)

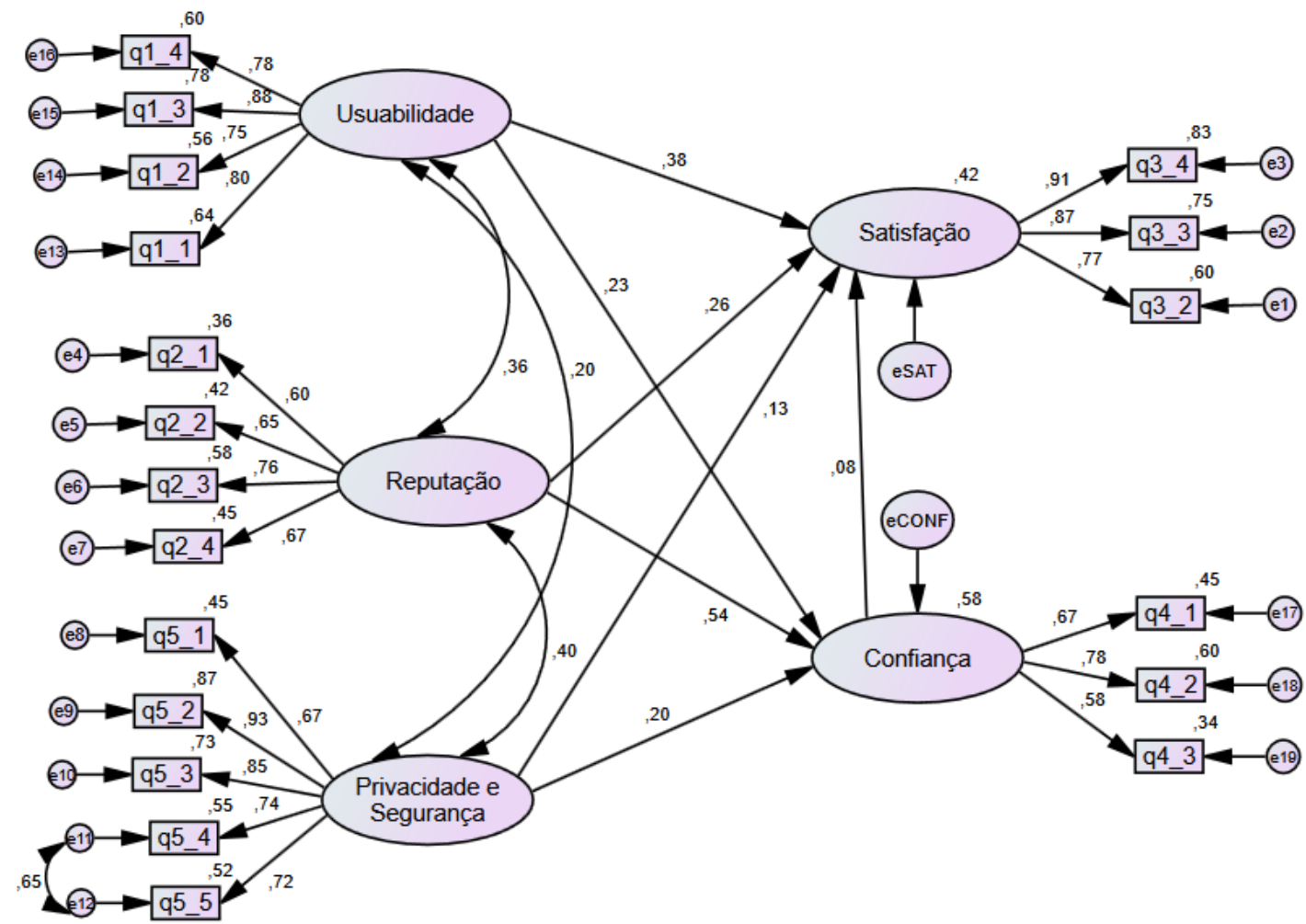

Nota. $x^{2} / g l=1,319 ; x^{2}=185,925 ; \mathrm{CFI}=0,978 ; \mathrm{RMSEA}=0,040 ; \mathrm{GFI}=0,915 ; \mathrm{TLI}=0,973 ; \mathrm{AGFI}=0,886$; $\mathrm{NFI}=0,915 ; \mathrm{PGFI}=0,679 ; \mathrm{PCFI}=0,806 ; \mathrm{SRMR}=0,0482$. Resultados significativos com valor-p<0,01. Fonte: Dados da pesquisa 2015, com o uso do AMOS v.20, utilizando a partição $B$ da amostra $(n=203)$. 
A Tabela 7 apresenta as hipóteses do Modelo de Equações Estruturais (MEE) que foram testadas. De fato, como proposto, a usabilidade, compreendida como a facilidade de navegação no website do internet banking, contribui positivamente para a satisfação do usuário (Hipótese 1). A usabilidade também está diretamente relacionada a confiança (Hipótese 2). A reputação, conceito diretamente associado à credibilidade percebida pelos consumidores, mostrou-se também como um fator que favorece a satisfação do usuário (Hipótese 4). A reputação também se mostrou um elemento que contribui bastante para o grau de confiança do usuário no internet banking (coeficiente padronizado de 0,522, (valor-p<0,001) (Hipótese 3). Os elementos Privacidade e Segurança resultaram também como importantes determinantes da satisfação e da confiança no internet banking. Na verdade, a percepção dos usuários relativas à segurança nos meios de pagamento e nos mecanismos de transmissão dos dados são necessárias para o desenvolvimento da confiança no ambiente virtual como a literatura tem proposto (Kolsaker e Payne, 2002; Gerrard et al., 2006; Susanto, Chang e Há, 2016) (Hipóteses 5 e 6). Arcand et al. (2017) também observaram que as dimensões da qualidade do serviço do mobile banking também influenciam a confiança e a satisfação. Por outro lado, os resultados rejeitam a hipótese 7 de que a confiança do usuário é um antecessor do grau de satisfação do mesmo. A regressão com a variável dependente Confiança apresentou $R^{2}=0,576$, o que confere um excelente nível de confiabilidade e a regressão para Satisfação obteve $R^{2}=0,420$, revelando uma boa confiabilidade. Ofori et al. (2017) testaram a hipótese de uma correlação positiva entre a confiança e a satisfação no serviço de internet banking e verificaram esta relação com o uso do PLS-SEM.

Tabela 7 - Verificação das hipóteses do modelo de equações estruturais

\begin{tabular}{lcccccc}
\hline \multicolumn{1}{c}{ Relação Estrutural } & $\begin{array}{c}\text { Coeficiente } \\
\text { padronizado }\end{array}$ & t-value & $\mathbf{R}^{\mathbf{2}}$ & Hipótese & Verificação \\
\hline Usabilidade $\rightarrow$ Satisfação & 0,382 & $4,850^{* * *}$ & 0,420 & $\mathrm{H} 1$ & confirmada \\
Usabilidade $\rightarrow$ Confiança & 0,230 & $2,889^{* * *}$ & 0,576 & $\mathrm{H} 2$ & confirmada \\
Reputação $\rightarrow$ Confiança & 0,540 & $4,917^{* * *}$ & 0,420 & $\mathrm{H} 3$ & confirmada \\
Reputação $\rightarrow$ Satisfação & 0,258 & $2,178^{* *}$ & 0,576 & $\mathrm{H} 4$ & confirmada \\
Privacidade/Segurança $\rightarrow$ Satisfação & 0,126 & $1,695^{*}$ & 0,420 & $\mathrm{H} 5$ & confirmada \\
Privacidade/Segurança $\rightarrow$ Confiança & 0,197 & $2,460^{* *}$ & 0,576 & $\mathrm{H} 6$ & confirmada \\
Confiança $\rightarrow$ Satisfação & 0,082 & 0,616 & 0,420 & $\mathrm{H} 7$ & Não confirmada \\
\hline
\end{tabular}

Nota: ***valor-p<0,01; **valor-p<0,05; *valor-p<0,10

Fonte: Dados da pesquisa 2015, com o uso da partição B $(n=203)$

\section{CONSIDERAÇÕES FINAIS}

A confiança e a satisfação têm importante papel nas estratégias de negócios via internet nos dias atuais nos quais novas formas de negociação e comunicação surgem a cada dia assim como cresce rapidamente o número de empresas disponibilizando serviços on-line como também o número de usuários que demandam estes serviços. Neste contexto, o serviço de internet banking tem destacado relevo devido à sua peculiaridade de relacionamento frequente entre empresa e cliente como também pelo fato de envolver montantes e transações que não são de conhecimento público. 
Este trabalho objetivou identificar quais atributos são relevantes para a geração de confiança e satisfação de usuários do internet banking. Especificamente, foi avaliada a influência de atributos apontados como relevantes pela literatura sobre os níveis de confiança e satisfação dos clientes do serviço internet banking: o padrão de usabilidade, a reputação e a privacidade/segurança. O modelo proposto foi testado por meio da técnica de Modelagem de Equações Estruturais.

Os construtos para confiança e satisfação aparecem nesse modelo como variáveis regressoras permitindo que seja observada a sua importância na prestação de serviços internet banking. Os resultados indicam que níveis mais elevados de usabilidade, reputação e privacidade/segurança trazidos pelos usuários do internet banking, de fato, contribuem para melhorar os níveis de confiança e satisfação dos usuários.

O nível de satisfação com a utilização do serviço online é positivamente influenciado pela usabilidade percebida. Outro resultado foi o fato da confiança do consumidor ser influenciada pela percepção de usabilidade no site. Assim, maiores níveis de usabilidade podem levar a níveis mais elevados de confiança do site. A forma com que as pessoas veem o serviço de internet banking, ao comparar a outros sites de bancos concorrentes oferecendo seus produtos e serviços, se relaciona positivamente com a confiança e a satisfação depositada na prestação do serviço. À medida que aumenta a segurança na transmissão das informações pessoais para o site e a privacidade dos usuários em relação a este serviço, aumenta o nível de confiança dos seus clientes.

Por fim, ressaltam-se como limitações desta pesquisa, alguns pontos que podem ser trabalhados em estudos futuros. No campo metodológico, a amostragem utilizada não permite uma generalização de seus resultados, algumas limitações também são observadas por fazer uso de dados de corte transversal, onde se sabe que o estudo longitudinal é mais apropriado para a determinação de relações de causa e efeito. Pesquisas futuras podem ser direcionadas a testar e avaliar outros atributos que sejam relevantes para a confiança e satisfação de serviços online de bancos comerciais.

\section{REFERÊNCIAS}

1. ARCAND, Manon et al. Mobile banking service quality and customer relationships. International Journal of Bank Marketing, v. 35, n. 7, p. 1068-1089, 2017.

2. BAILEY, J.; PEARSON, S. Development of a tool for measurement and analyzing computer user satisfaction. Management Science, 29(5), pp. 530 -575, 1983.

3. BAUER, H. H.; GRETHER, M.; LEACH, M. Building Customer Relations Over the Internet. Industrial Marketing Management, 31, pp. 155-163, 2002.

4. BART, Y.; SHANKAR, V.; SULTAN, F.; URBAN, G. L. Are the Drivers and Role of Online Trust the Same for All Web Sites and Consumers? A Large-Scale Exploratory Empirical Study. Journal of Marketing, 69, pp. 133-152, oct. 2005.

5. BERRY, L. Relationship marketing of services - perspectives from 1983 and 2000. Journal of Relationship Marketing, 1 (1), pp. 59-77, 2002. 
6. BREI, V. A.; ROSSI, C. A. V. Confiança, Valor Percebido e Lealdade em Trocas Relacionadas de Serviço: Um Estudo com Usuários de Internet Banking no Brasil. RAC, 9 (2), pp. 145-168, 2005.

7. BUTT, M. M.; AFTAB, M. Incorporating attitude towards Halal banking in an integrated service quality, satisfaction, trust and loyalty model in online Islamic banking context. International Journal of Bank Marketing, 31(1), pp. 6-23, 2013.

8. CASALÓ, L.; CARLOS, F.; GUINALÍU, M. The Generation of Trust in the Online Services and Product Distribution: The case of Spanish Electronic Commerce. Journal of Electronic Commerce Research, 12 (3), pp. 199-213, 2011.

9. CORRITORE, C., KRACHER, B; WIEDENBECK, S. On-Line Trust: Concepts, Evolving Themes, a Model. International Journal of Human-Computer Studies, 58 (6), pp. 737-758, 2003.

10. ENNEW, C.; SEKHON, H. Measuring trust in financial services: the Trust Index. Conxumer Policy Review, 17 (2), mar./apr. 2007.

11. FÁVERO, L. P.; BELFIORE, P.; SILVA, F. L.; CHAN, B. L. Análise de dados: modelagem multivariada para tomada de decisões. 1 ed. Rio de Janeiro: Elsevier, 646p, 2009.

12. FEBRABAN, “Pesquisa FEBRABAN de Tecnologia Bancária 2019”, 2019. Disponível em: < https://portal.febraban.org.br/pagina/3106/48/pt-br/pesquisa > Acesso em 27/08/2019.

13. FLAVIÁN. C.; M. GUINALÍU. Consumer Trust, Perceived Security, and Privacy Policy: Three Basic Elements of Loyalty to a Website. Industrial Management and Data Systems, 106 (5), pp. 601-620, 2006.

14. GEORGE, A.; KUMAR, G. S. G. Validation of a scale for measuring problems in internet banking and their effect on customer satisfaction. Vision: The Journal of Business Perspective. Vol. 19, no 4, pp. 312-323, dec. 2015.

15. GERRARD, P.; CUNNINGHAM, J. B.; DEVLIN, J. F. Why consumers are not using internet banking: a qualitative study. Journal of Services Marketing, 20 (3), pp. 160-168, 2006.

16. GIOVANNINI, C. J.; NUNES, D. C. G.; FERREIRA, D. B. Satisfação e Confiança do Consumidor em Ambientes Virtuais de Corretoras de Valores. Anais do XXXVI Encontro da ANPAD, Rio de Janeiro, 2012.

17. GRÖNROOS, C. Service management and marketing: a customer relationship management approach. 2 ed. England: Wiley, p. 394, 2000.

18. GUPTA, K. K.; BANSAL, I. Development of an Instrument to Measure Internet Banking Service Quality in India. Journal os Arts, Science \& Commerce, 3 (2), apr. 2012.

19. GUTMAN, L. F. D.; JOIA, L. A.; MORENO JR., V. A. Antecedentes da intenção de uso de sistemas de home broker sob a ótica dos investidores do mercado acionário. Revista de Administração, 49 (2), pp. 353-368, 2014.

20. HAIR, J. F.; TATHAM, R. L.; ANDERSON, R. E.; BLACK, W. Análise multivariada de dados. 6.ed. Porto Alegre: Bookman, 688p, 2009. 
21. HANSON, W. Principles of internet marketing. Cincinnati: South-Western College Publishing, 2000.

22. HERNANDEZ, J. M. D. C.; MAZZON, J. A. Um estudo empírico dos determinantes da adoção de Internet Banking entre não usuários brasileiros. Revista de Administração Contemporânea, 12, p. 9-39, 2008.

23. IBRAHIM, E. N. M.; NOOR, N. L. M; MEHAD, S. Trust or distrust in the web-mediated information environment (W-MIE): A perspective of online Muslim users. Journal os Enterprise Information Management, 22(5), pp. 523-547, 2009.

24. IVES, B., OLSON, M.; BAROUDI, S. The measurement of user information satisfaction. Communications of the ACM, 26(10), pp. 785-793, 1983.

25. JARVENPAA, S. L.; TRACTINSKY, N.; VITALE, M. Consumer trust in an internet store. Information Technology and Management, 1 (1), pp. 45-71, 2000.

26. JOSANG, A.; ISMAIL, R.; BOYD, C. A Survey of Trust and Reputation Systems for Online Service Provision. Decision Support Systems, 43 (2), pp. 618-644, 2007.

27. KANTOWITZ, B. H.; HANKOWSKI, R. J.; KANTOWITZ, S. C. Driver Acceptance of Unreliable Traffic Information in Familiar and Unfamiliar Settings. Human Factors, 39 (2), pp. 164-176, 1997.

28. KESHARWANI, A.; BISHT, S. S. The impact of trust and perceived risk on internet banking adoption in India: An extension of technology acceptance model. International Journal of Bank Marketing, 30 (4), pp. 303-322, 2012.

29. KHAN, Imran; RAHMAN, Zillur. Brand experience and emotional attachment in services: The moderating role of gender. Service Science, v. 9, n. 1, p. 50-61, 2017.

30. KLINE, P. The handbook of psychological testing. Routledge: London, 2000.

31. KLINE, R.B. Principles and practice of structural equation modeling. New York London: The Guilford Press. 2 ed, 366p, 2005.

32. KOLSAKER, A.; PAYNE, C. Engendering Trust in e-Commerce: A Study of Gender-based Concerns. Marketing Intelligence and Planning, 20 (4), pp. 206-214, 2002.

33. KOMIAK, S. X.; BENBASAT, I. Understanding Customer Trust in Agent-Mediated Electronic Commerce, Web-Mediated Eletronic Commerce, and Traditional Commerce. Information Technology and Management, 5 (1), pp. 181-207, jan. 2004.

34. LEE, N.; HOOLEY. G. The evolution of "classical mythology" within marketing measure development. European Journal of Marketing, 39 (3/4), pp. 365- 385, 2005.

35. LI, Y. Empirical studies on online information privacy concerns: literature review and an integrative framework. Communications of the Association for Information Systems, [S.I.], v. 28, n. 1, p. 453-496, 2011.

36. LIAO, Z.; CHEUNG, M. T. Measuring consumer satisfaction in internet banking: a core framework. Communications of the ACM, 51 (4), pp. 47-51, 2008. 
37. LIAO, C.; LIU, C. C.; CHIEN, K. Examining the impact of privacy, trust and risk perceptions beyond monetary transactions: an integrated model. Electronic Commerce Research and Applications, [S.I.], v. 10, n. 6, p. 702-715, 2011.

38. LIÉBANA-CABANILLAS, F.; MUÑOZ-LEIVA, F.; REJÓN-GUARDIA, F. The determinants of satisfaction with e-banking. Industrial Management \& Data Systems, 113(5), pp. 750-767, 2013

39. LOEBBECKE, C.; HORNIG, V. Increasing trust in e-commerce: concepts and examples of insurance solutions. In: $14^{\mathrm{TH}}$ Bled Electronic Commerce Conference. Bled, Slovenia. June. 2001.

40. MA, Z. Assessing Serviceability and Reliability to Affect Customer Satisfaction of Internet Banking. Journal of Software, 7 (7), 2012.

41. MARÔCO, J. Análise de Equações Estruturais: fundamentos teóricos, software \& aplicações. 1a ed. Lisboa - Portugal, ReportNumber, 2010.

42. MARÔCO, J. Análise Estatística com o SPSS Statistics. 5a ed. Lisboa - Portugal, ReportNumber, 2011.

43. MELONE, N.P. A theoretical assessment of the user satisfaction construct in information systems research. Management Science, pp. 76-91, 1990.

44. MORGAN, R.; HUNT, S. The Commitment-Trust Theory of Relationship Marketing. Journal of Marketing, 58 (3), pp. 20-38, Jul. 1994.

MORGAN-THOMAS, A.; VELOUTSOU, C. Beyond technology acceptance: Brand relationships and online brand experience. Journal of Business Research, 66 (1), pp. 21-27, 2013.

45. MOORMAN, C.; DESHPANDÉ, R.; ZALTMAN, G. Factors Affecting Trust in Market Research Relationships. Journal of Marketing, 57 (1), pp. 81-101, Jan. 1993.

46. MOUTINHO, L.; SMIT, A. Modelling bank customer satisfaction through mediation of attitudes towards human and automated banking. International Journal of Bank Marketing, 18 (3), pp. 124-134, 2000.

47. MUIR, B. M.; MORAY, N. Trust in automation: Part $H$ Experimental studies of (rust and human intervention in a process control simulation. Ergonomics, 39 (3), pp. 429-461, 1996.

48. OFORI, Kwame Simpe et al. Examining customers' continuance intentions towards internet banking usage. Marketing Intelligence \& Planning, v. 35, n. 6, p. 756-773, 2017.

49. O'MALLEY, L.; TYNAN, C. Relationship marketing in consumer markets - rhetoric or reality? European Journal of Marketing, 34(7), pp. 797-815, 2000.

50. POlATOGLU, V. N.; EKIN, S. An empirical investigation of the Turkish consumers' acceptance of internet banking services. International Journal of Bank Marketing, 19 (4), pp. 156-165, 2001.

51. PRADO, E. P. V. Sites de Internet Banking: Uma Avaliação da Qualidade Baseada no Modelo Webqual. Gestão \& Regionalidade. 26 (77), 2010. 
52. RIQUELME, H. E.; MEKKAOUI, K. A.; RIOS, R. E. Internet Banking Customer Satisfaction and Online Service Attributes. Journal of Internet Banking and Commerce, 14 (2), 2009.

53. ROUSSEAU, D. M.; SITKIN, S. B.; BURT, R. S.; CAMERER, C. Not so Different After All: A Cross Discipline View of Trust. The Academy of Management Review, 23 (3), pp. 393-404, Jul. 1998.

54. ROOSTIKA, RATNA Perceived service quality and trust on satisfaction: customer's perspectives in the banking sector. Jurnal Keuangan dan Perbankan, 15(2), pp. 271-280, 2011.

55. SAFEENA, R.; KAMMANI, A.; DATE, H. Assessment of Internet Banking Adoption: An Empirical Analysis. Arabian Journal for Science and Engineering, 39 (2), p. 837-849, 2014.

56. SANTOS, T. S.; CONDE, D. R.; SANTOS, T. S.; ANTONIALLI, L. M. Internet Banking: perfil do usuário e qualidade percebida pelos clientes bancários de Lavras/MG. Anais do III Encontro de Administração da Informação, Porto Alegre, 2011.

57. SEDDON, P. B. A Respecification and Extension of the DeLone and McLean Model of IS Success. Information Systems Research, 8 (3), pp. 240-253, 1997.

58. SELNES, F. Antecedents and Consequences of Trust and Satisfaction in Buyer-Seller Relationships. European Journal of Marketing, 32 (3), pp. 305-322, 1998.

59. SIKDAR, P.; KUMAR, A.; MAKKAD, M. Online Banking Adoption: A Factor Validation and Satisfaction Causation Study in the Context of Indian Banking Customers. International Journal of Bank Marketing, 33 (6), p. 760-785, 2015.

60. SUSANTO, A.; CHANG, Y.; HA, Y. Determinants of continuance intention to use the smartphone banking services: An extension to the expectation-confirmation model. Industrial Management \& Data Systems, 116 (3), pp. 508-525, 2016.

61. TWUM, F.; AHENKORA, K. Internet Banking Security Strategy: Securing Customer Trust. Journal of Management and Strategy, 3 (4), pp. 78-83, 2012.

62. VIACAVA, J. J. C.; MANTOVANI, D.; KORELO, J. C.; PRADO, P. H. M. Vieses na satisfação: o efeito moderador da dificuldade e da obrigação sobre recordações positivas e negativas. Revista Eletrônica de Administração, 81 (2), pp. 437-461, 2015.

63. WIXOM, B. H.; TODD, P. A. A theoretical integration of user satisfaction and technology acceptance. Information Systems Research, 16(1), pp. 85-102, 2005.

64. YOUSAFZAI, S. Y.; FOXALL, G. R.; PALLISTER, J. G. Explaining internet banking behavior: theory of reasoned action, theory of planned behavior, or technology acceptance model? Journal of Applied Social Psychology, 40 (5), pp. 1172-1202, 2010.

65. YU, P. L.; BALAJI, M. S.; KHONG, K. W. Building trust in internet banking: a trustworthiness perspective. Industrial Management \& Data Systems, 115 (2), pp. 235-252, 2015.

66. ZHAO, A. L.; LEWIS, N. K.; HANMER-LLOYD, S. \& WARD, P. Adoption of internet banking services in China: is it all about trust? International Journal of Bank Marketing, 28 (1), pp. 726, 2010. 
67. ZHU, Y.; CHEN, H. Service fairness and customer satisfaction in internet banking. Internet Research, 22 (4), pp. 482-498, 2012. 